Pacific Journal of Mathematics

ON THE ADDITIVITY OF LATTICE COMPLETENESS 


\section{ON THE ADDITIVITY OF LATTICE COMPLETENESS}

to the memory of Maurice Audin

ISRAEL HALPERIN AND MARIA WONENBURger

1. Introduction. It was shown in [1, Theorem 4.3] that upper $\boldsymbol{\aleph}$ continuity $^{1}$ is additive in the following sense:

(1.1) Suppose that $[0, a],[0, b]$ are upper $\$$-continuous in a relatively complemented modular lattice. Then $[0, a \cup b]$ is upper $\$$-continuous provided that $[0, a \cup b]$ is upper $\aleph$-complete.

But it may happen that $[0, a],[0, b]$ are both upper $\boldsymbol{K}$-complete (both may even be von Neumann geometries with a perspective to $b$ ) and yet $[0, a \cup b]$ is not upper $\aleph$-complete. In fact there are von Neumann rings $\mathscr{R}$ for which the lattice $\bar{R}_{\mathscr{S}}$, with $\mathscr{S}=\mathscr{R}_{2}$, is not even upper $\aleph_{0}$-complete (see the Remark preceding Definition 3.1)

With a modest supplementary condition however, additivity of upper $\$$-completeness does hold, as we show in this paper.

2. Terminology and notation. We shall use the notation of [1], [2], and [4].

I will denote a set of indices $\alpha$ and $\bar{I}$ will denote the cardinal power of $I$.

$\$$ will denote an infinite cardinal, $\Omega$ will denote the least ordinal number whose corresponding cardinal power is $\boldsymbol{\aleph}$.

A lattice is called upper $\$$-complete if the union $a=\mathbf{U}\left(a_{\alpha} \mid \alpha \in I\right)$ exists whenever $\bar{I} \leqq \boldsymbol{K}$, and is called upper $\boldsymbol{K}$-continuous if for every $b$ : $b \cap a=\bigcup\left(\left(b \cap \bigcup\left(a_{\alpha} \mid \alpha \in F\right)\right) \mid\right.$ all finite $\left.F \subset I\right)$, with dual definitions for lower $\boldsymbol{\aleph}$-completeness and lower $\$$-continuity. The lattice is called $\aleph$ complete, respectively $\boldsymbol{\aleph}$-continuous if it is both upper and lower Si-continuous.

A complemented modular lattice $L$ is called an $\aleph$-von Neumanngeometry if it is $\boldsymbol{\aleph}$-complete and $\boldsymbol{K}$-continuous (irreducibility is not assumed).

If we omit the $\aleph$ in any of these designations, this implies that the lattice $L$ has the corresponding $\boldsymbol{\aleph}$-property for all $\boldsymbol{\aleph}$.

If $\mathscr{R}$ is an associative regular ring (not necessarily with unit element) then $\bar{R}_{\mathscr{R}}$ denotes the relatively complemented modular lattice of its principal right ideals, ordered by inclusion. $\mathscr{R}$ is called an $\$$-von Neumannring, respectively a von Neumann ring, according as $\bar{R}_{\mathscr{S}}$ is an $\boldsymbol{K}$-von

Received December 28, 1961. Dr. Wonenburger is a postdoctorate Fellow (of the National Research Council of Canada) at Queen's University.

1 Terminology and notation are explained in section 2 below. 
Neumann-geometry, respectively a von Neumann geometry.

In any relatively complemented modular lattice, if $a \geqq b$ then $[a-b]$ will denote an arbitrary (but fixed) element such that $[a-b] \dot{U} b=a$ (the dot indicates that the summands in the union are independent). We write $a \sim b$ to denote: $a$ is perspective to $b$, and $a \lesssim b$ to denote: $a \sim b_{1}$ for some $b_{1} \leqq b$. Elements $a, b$ are called completely disjoint, (notation: $(a, b) P)$ if: $a_{1} \sim b_{1}, a_{1} \leqq a, b_{1} \leqq b$ together imply $a_{1}=0$.

3. The additivity of completeness theorem.

In this section $a, b, c, \cdots x_{\alpha}, \cdots$ will denote elements in a given relatively complemented modular lattice $L$.

If $[0, a \cup c]$ is upper $\boldsymbol{\aleph}$-complete we shall write $u(a, c, \mathfrak{\aleph})$ to mean:

$$
\text { Whenever } \left.x_{a} \leqq a \cup c \text { for all } \alpha \in I \text { (with } \bar{I} \leqq \aleph\right) \text { and }
$$

$$
a \cap\left(\bigcup\left(x_{\beta} \mid \beta \in F\right)\right)=0
$$

for all finite $F \subset I$, then $a \cap\left(\bigcup\left(x_{\alpha} \mid \alpha \in I\right)\right)=0$.

It is important to note: if $u(a, c, \boldsymbol{\aleph})$ holds then $u\left(a^{\prime}, c^{\prime}, \boldsymbol{\aleph}\right)$ holds for all $a^{\prime} \leqq a, c^{\prime} \leqq c$.

Clearly, if $[0, a \cup c]$ is upper $\$$-complete and upper $\$$-continuous then $u(a, c, \mathbf{W})$ does hold.

Similarly, if $[0, a \cup c]$ is lower $\$$-complete we shall write $l(a, c, \aleph)$ to denote:

$\overline{(3.1)} \quad$ Whenever $x_{\alpha} \leqq a \cup c$ for all $\alpha \in I$ (with $\left.\bar{I} \leqq \aleph\right)$ and

$$
a \cup\left(\cap\left(x_{\beta} \mid \beta \in F\right)\right)=a \cup c
$$

for all finite $F \subset I$, then $a \cup\left(\bigcap\left(x_{\alpha} \mid \alpha \in I\right)\right)=a \cup c$.

It is important to note: if $l(a, c, \boldsymbol{W})$ holds then $l\left(a^{\prime}, c^{\prime}, \boldsymbol{W}\right)$ holds for all $a^{\prime} \leqq a, c^{\prime} \leqq c$.

Clearly, if $[0, a \cup c]$ is lower $\$$-complete and lower $\$$-continuous then $l(a, c, \aleph)$ does hold.

LEMma 3.1. Suppose that each of $[0, a \cup b],[0, b \cup c],[0, a \cup c]$ is upper $\aleph$-complete and suppose that $u(a, c, \aleph)$ holds. Then $[0, a \cup b \cup c]$ is upper $\aleph$-complete.

Proof. We may suppose that $\{a, b, c\}$ is an independent set, for if $c, b$ are replaced by $[c-(a \cap c)]$ and $[b-(b \cap(a \cup c))]$ respectively the hypotheses of Lemma 3.1 continue to hold and the conclusion is not changed.

Using transfinite induction, we may suppose that Lemma 3.1 holds 
for all $\boldsymbol{\aleph}^{\prime}<\boldsymbol{\aleph}$. We may therefore assume that $x_{a}$ is given, $\leqq a \cup b \cup c$ for all $0<\alpha<\Omega$, that $\mathrm{U}\left(x_{\alpha} \mid \alpha \leqq \beta\right)$ exists for all $\beta<\Omega$ and we need only show that $\bigcup\left(x_{\alpha} \mid \alpha<\Omega\right)$ exists.

We may suppose $x_{\alpha} \leqq x_{\beta}$ for $\alpha \leqq \beta<\Omega$ (by replacing the original $x_{\alpha}$ by $\mathrm{U}\left(x_{\beta} \mid \beta \leqq \alpha\right)$ for all $(\alpha<\Omega)$.

Set $\bar{x}_{0}=\mathbf{U}\left(\left(x_{\alpha} \cap(a \cap b)\right) \mid \alpha<\Omega\right)$ (this union exists since, by hypothesis, $[0, a \cup b]$ is upper $\aleph$-complete). Set $\bar{x}_{\alpha}=\bar{x}_{0} \cup x_{\alpha}$ for $0<\alpha<\Omega$ and observe that $\bar{x}_{\beta} \leqq \bar{x}_{\alpha}$ for all $0 \leqq \beta \leqq \alpha<\Omega$.

Set $y_{0}=\bar{x}_{0}$ and $y_{\alpha}=\left[\bar{x}_{\alpha}-\bigcup\left(\bar{x}_{\beta} \mid 0 \leqq \beta<\alpha\right)\right]$ for $0<\alpha<\Omega$. Then $\mathrm{U}\left(y_{\beta} \mid 0 \leqq \beta<\alpha\right)=\mathrm{U}\left(\bar{x}_{\beta} \mid 0 \leqq \beta<\alpha\right)$ for all $0<\alpha<\Omega$, as may be verified easily by transfinite induction.

Clearly, we need only show that $\mathrm{U}\left(y_{\alpha} \mid 0 \leqq \alpha<\Omega\right)$ exists. Hence it is sufficient to show that $\mathrm{U}_{\alpha} y_{\alpha}$ exists, where (for the rest of this proof) we write $\mathrm{U}_{\alpha}$ to mean $\mathrm{U}_{0<\alpha<\Omega}$ (note: $0 \leqq \alpha<\Omega$ has been replaced by $0<\alpha<\Omega)$.

Set $u=\left(a \cup\left(\bigcup_{\alpha}\left(\left(a \cup y_{\alpha}\right) \cap(b \cup c)\right)\right)\right) \cap\left(b \cup\left(\bigcup_{a}\left(\left(b \cup y_{a}\right) \cap(a \cup c)\right)\right)\right)$ (this union exists since, by hypothesis, $[0, b \cup c]$ and $[0, a \cup c]$ are upper ऊ-complete). We observe that $u \geqq y_{\beta}$ for all $0<\beta<\Omega$ since each factor of $u$ has this property: for fixed $\beta, a \cup\left(\bigcup_{\alpha}\left(\left(a \cup y_{\alpha}\right) \cap(b \cup c)\right)\right) \geqq$ $a \cup\left(\left(a \cup y_{\beta}\right) \cap(b \cup c)\right)=\left(a \cup y_{\beta}\right) \cap(a \cup b \cup c)=a \cup y_{\beta} \geqq y_{\beta}$.

We shall show that $u$ is the desired union $\mathrm{U}_{\alpha} y_{\alpha}$. It is clearly sufficient to show for every $w$ : if $u \geqq w \geqq y_{\alpha}$ for all $0<\alpha<\Omega$ then $u \leqq w$.

Since $a \cup y_{\alpha} \leqq a \cup w$ and $b \cup y_{\alpha} \leqq b \cup w$ for all $0<\alpha<\Omega$,

$$
\begin{aligned}
u & \leqq(a \cup((a \cup w) \cap(b \cup c))) \cap(b \cup((b \cup w) \cap(a \cup c))) \\
& =(a \cup w) \cap(b \cup w)=w \cup(a \cap(b \cup w)) .
\end{aligned}
$$

It is therefore sufficient to show that $a \cap(b \cup w) \leqq w$. We shall show that $a \cap(b \cup u)=0$; this will imply:

$$
a \cap(b \cup w) \leqq a \cap(b \cup u)=0 \leqq w .
$$

Now $\left.b \cup u=\left(a \cup b \cup\left(\bigcup_{\alpha}\left(a \cup y_{\alpha}\right) \cap(b \cup c)\right)\right)\right) \cap\left(b \cup\left(\bigcup_{\alpha}\left(\left(b \cup y_{\alpha}\right) \cap(a \cup c)\right)\right)\right)$,

$$
\begin{aligned}
a \cap(b \cup u) & =a \cap\left(b \cup\left(\bigcup_{\alpha}\left(\left(b \cup y_{\alpha}\right) \cap(a \cup c)\right)\right)\right) \\
& =a \cap\left((b \cap(a \cup c)) \cup\left(\bigcup_{\alpha}\left(\left(b \cup y_{\alpha}\right) \cap(a \cup c)\right)\right)\right) \\
& =a \cap\left(\bigcup_{\alpha}\left(\left(b \cup y_{\alpha}\right) \cap(a \cup c)\right)\right) .
\end{aligned}
$$

Since $u(\alpha, c, \boldsymbol{W})$ is assumed to hold we need only show:

$$
a \cap\left(\bigcup\left(\left(\left(b \cup y_{\alpha}\right) \cap(a \cup c)\right) \mid \alpha=\alpha_{1}, \cdots, \alpha_{m}\right)\right)=0
$$

for every finite set of indices $0<\alpha_{1}<\alpha_{2}<\cdots<\alpha_{m}<\Omega$.

Hence it is sufficient to show that

$$
a \cap\left(b \cup\left(\bigcup\left(y_{\alpha} \mid \alpha=\alpha_{1}, \cdots, \alpha_{m}\right)\right)\right)=0,
$$


and so it is sufficient to show that

$$
(a \cup b) \cap\left(\bigcup\left(y_{\alpha} \mid \alpha=\alpha_{1}, \cdots, \alpha_{m}\right)\right)=0 .
$$

For this purpose, we note: $y_{\alpha} \cap\left(\mathrm{U}\left(y_{\beta} \mid 0 \leqq \beta<\alpha\right)=0\right.$ for all $0<\alpha<\Omega$. This implies that $\left\{y_{\alpha} \mid \alpha=0, \alpha_{1}, \cdots, \alpha_{m}\right\}$ is an independent set and hence $y_{0} \cap\left(\mathrm{U}\left(y_{\alpha} \mid \alpha=\alpha_{1}, \cdots, \alpha_{m}\right)\right)=0$. This implies (3.2) since the left side of (3.2) is $\leqq y_{0}$. Thus Lemma 3.1 is proved.

CoRollary 1. Suppose that $\left[0, a_{i} \cup a_{j}\right]$ is upper $\aleph$-complete for $i, j=1, \cdots, m$ for some finite integer $m$ and suppose that $u\left(a_{i}, a_{j}, \boldsymbol{\aleph}\right)$ holds whenever $i<j$. Then $\left[0, a_{1} \cup \cdots \cup a_{m}\right]$ is upper $\aleph$-complete.

Proof. If $m \leqq 2$ the conclusion is part of the hypotheses. Suppose that $m>2$ and that the Corollary is known to hold with $m-1$ in place of $m$; then Lemma 3.1 can be applied (with $a=a_{1}, b=a_{3} \cup \cdots \cup a_{m}$ and $c=a_{2}$ ) to show that the Corollary holds for $m$ itself. By induction on $m$, the Corollary is established.

CoRollary 2. Suppose that $\left[0, a_{i} \cup a_{j}\right]$ is upper $\aleph$-complete and upper $\aleph$-continuous for $i, j=1, \cdots, m$ for some finite integer $m$. Then $\left[0, a_{1} \cup \cdots \cup a_{m}\right]$ is upper $\aleph$-complete and upper $\aleph$-continuous.

Proof. Since upper $\$$-continuity of $\left[0, a_{i} \cup a_{j}\right]$ implies that $u\left(a_{i}, a_{j}, \aleph\right)$ holds, Corollary 1 shows that $\left[0, a_{1} \cup \cdots \cup a_{m}\right]$ is upper $\$$-complete. The upper $\$$-continuity then follows from [1, Theorem 4.3].

LEMMA 3.2. Suppose that $a=a_{1} \cup a_{2} \cup \cdots \cup a_{m}$ and $a_{i} \lesssim a_{1} \cup \cdots \cup a_{i-1}$ for $1<i \leqq m$. Then a can be expressed in the form:

(3.3) $a_{1} \dot{\cup} \bar{a}_{2} \dot{\cup} \cdots \dot{\cup} \bar{a}_{n}$ for some $n \geqq m$ and elements $\bar{a}_{2}, \cdots, \bar{a}_{n}$ such that $\bar{a}_{i} \lesssim a_{1}$ for all $1<i \leqq n$.

Moreover $\bar{\alpha}_{2}$ may be taken to coincide with $a_{2}$ if $a_{1} \cap a_{2}=0$.

Proof. Lemma 3.2 holds trivially if $m=1$ and also if $m=2$ and $a_{1} \cap a_{2}=0$. We may therefore suppose (by induction) that $m>1$ and that $b=a_{1} \cup \cdots \cup a_{m-1}$ has the form (3.3).

We can replace $a_{m}$ by $\left[a_{m}-\left(a_{m} \cap b\right)\right]$ since the hypotheses of Lemma 3.2 continue to hold and the conclusion is not changed. After this change,

$$
a_{m} \cap b=a_{m} \cap\left(a_{1} \dot{\cup} \bar{a}_{2} \dot{\cup} \cdots \dot{\cup} \bar{a}_{n}\right)=0 .
$$

Since $a_{m} \lesssim a_{1} \dot{\cup} \bar{a}_{2} \dot{U} \cdots \dot{U} \bar{a}_{n}$ there is a perspectivity mapping $\varphi$ of $\left[0, a_{m}\right]$ with $\varphi\left(a_{m}\right) \leqq b$. Then

$$
a_{m}=a_{m, 1} \dot{\cup} a_{m, 2} \dot{\cup} \cdots \dot{\cup} a_{m, n}
$$


where

$$
\varphi\left(a_{m, 1}\right)=\varphi\left(a_{m}\right) \cap a_{1}
$$

and for $1<i \leqq n$,

$$
\begin{aligned}
\varphi\left(a_{m, i}\right) & =\left[\left(\varphi\left(a_{m}\right) \cap\left(a_{1} \dot{U} \bar{a}_{2} \dot{U} \cdots \dot{U} \bar{a}_{i}\right)\right)\right. \\
& -\left(\varphi\left(a_{m}\right) \cap\left(a_{1} \dot{\cup} \bar{a}_{2} \dot{U} \cdots \dot{U} \bar{a}_{i-1}\right)\right] .
\end{aligned}
$$

Obviously, $a_{m, 1} \lesssim a_{1}$. If $i>1$ then $a_{m, i} \sim \varphi\left(a_{m, i}\right) ; \varphi\left(a_{m, i}\right) \lesssim \bar{a}_{i} ; \bar{a}_{i} \lesssim a_{1}$; and $a_{m, i} \cap\left(\varphi\left(a_{m, i}\right) \cup \bar{a}_{i} \cup a_{1}\right)=0$; these facts imply that $a_{m, i} \lesssim a_{1}$ (use (2.2) of [1]). The conclusion of Lemma 3.2 now follows at once.

\section{Lemma 3.3. Suppose that}

(i) $a=a_{1} \cup a_{2} \cup \cdots \cup a_{m}$ for some finite $m \geqq 2$,

(ii) $a_{2} \sim a_{1}$,

(iii) $a_{i} \lesssim a_{1} \cup \cdots \cup a_{i-1}$ for $2<i \leqq m$,

(iv) $\left[0, a_{1} \cup a_{2}\right]$ is upper $\aleph$-complete,

(v) $u\left(a_{1}, a_{2}, \boldsymbol{W}\right)$ holds.

Then $[0, a]$ is upper $\$$-complete.

Proof. Applying Lemma 3.2, and using a new $m$ and new elements $a_{3}, \cdots, a_{m}$ we may suppose that (i), (iii) hold in the strengthened form: $a=a_{1} \dot{\cup} a_{2} \dot{U} \cdots \dot{\cup} a_{m}$ and $a_{i} \lesssim a_{1}$ for $2<i \leqq m$.

Suppose that $1 \leqq i<j \leqq m$. If $i \neq 2$ then $a_{j} \lesssim a_{2}$ (because of (ii)) and there is a perspectivity mapping $\varphi$ of $\left[0, a_{i} \cup a_{j}\right]$ with $\varphi\left(a_{i}\right) \leqq a_{1}$ and $\varphi\left(a_{j}\right) \leqq a_{2}$. Hence $\left[0, a_{i} \cup a_{j}\right]$ is upper $\boldsymbol{\aleph}$-complete and $u\left(a_{i}, a_{j}, \boldsymbol{\aleph}\right)$ holds in this case.

If $i=2$ there is a perspectivity mapping $\varphi$ of $\left[0, a_{2} \cup a_{j}\right]$ with $\varphi\left(a_{2}\right)=$ $a_{1}, \varphi\left(a_{j}\right)=a_{j}$; the result for $\left[0, a_{1} \cup a_{j}\right]$ obtained previously now implies: $\left[0, a_{2} \cup a_{j}\right]$ is upper $\boldsymbol{\aleph}$-complete and $u\left(a_{2}, a_{j}, \boldsymbol{\aleph}\right)$ holds.

Corollary 1 to Lemma 3.1 now applies to these elements $a_{1}, \cdots, a_{m}$ and this completes the proof of Lemma 3.3.

Corollary. Suppose that the hypotheses (i), (ii), (iii), of Lemmx 3.3 hold and suppose also that

(vi) $\left[0, a_{1} \dot{\cup} a_{2}\right]$ is upper $\aleph$-complete and upper $\aleph$-continuous.

Then $[0, a]$ is upper $\mathbf{S}$-complete and upper $\mathbf{K}$-continuous.

Proof. (vi) implies (iv), (v). Hence $[0, a]$ is upper $\$$-complete by Lemma 3.3. Upper $\aleph$-continuity then follows from [1, Theorem 4.3].

Lemma 3.4. (Additivity of lower $\aleph$-continuity). Suppose that $\left[0, a_{1} \cup \cdots \cup a_{m}\right]$ is lower $\aleph$-complete and that $\left[0, a_{i}\right]$ is lower $\leftrightarrow$ - 
continuous for $i=1, \cdots, m$. Then $\left[0, a_{1} \cup \cdots \cup a_{m}\right]$ is lower $\aleph$-continuous.

Proof. We may assume that $\left\{a_{1}, \cdots, a_{m}\right\}$ is an independent set (replace $a_{i}$ by $\left[a_{i}-\left(a_{i} \cap\left(a_{1} \cup \cdots \cup a_{i-1}\right)\right)\right]$ for $\left.2 \leqq i \leqq m\right)$.

Then $\left[a_{1}, a_{1} \cup a_{2}\right]$ is lower $\boldsymbol{K}$-continuous since it is lattice isomorphic to $\left[0, a_{2}\right]$ under the mapping: $x \rightarrow x \cap a_{2}$. Similarly $\left[a_{2}, a_{1} \cup a_{2}\right]$ is lower S-continuous. By the dual of [1, Theorem 4.3], $\left[0, a_{1} \cup a_{2}\right]=\left(\left[a_{1} \cap a_{2}\right.\right.$, $\left.a_{1} \cup a_{2}\right]$ ) is lower $\aleph$-continuous. Lemma 3.4 follows by induction on $m$.

Lemma 3.5. Suppose that each of $[0, a \cup b],[0, b \cup c),[0, a \cup c]$ is lower $\aleph$-complete and suppose that $l(a, c, \aleph)$ holds. Then $[0, a \cup b \cup c]$ is lower $\aleph$-complete.

Proof. We may suppose that $\{a, b, c\}$ is an independent set, for if $c, b$ are replaced by $[c-(a \cap c)]$ and $[b-(b \cap(a \cup c))]$ respectively the hypotheses of Lemma 3.5 continue to hold $\left(l\left(a, c_{1}, \aleph\right)\right.$ is equivalent to. $l(a, c, \aleph)$ if $\left.a \cup c_{1}=a \cup c\right)$ and the conclusion is not changed.

Now set $B=a \cup c, C=b \cup a, A=b \cup c$, and $1=a \cup b \cup c$. We have: $[A \cap B, 1](=[c, a \cup b \cup c])$ is lower $\aleph$-complete since it is lattice isomorphic to $[0, a \cup b]$ under the mapping $x \rightarrow x \cap(a \cup b)$. Similarly each of $[B \cap C, 1],[C \cap A, 1]$ is lower $\$$-complete.

We can now show that $[0, a \cup b \cup c](=[A \cap B \cap C, 1])$ is lower $\boldsymbol{\aleph}$ complete (by applying the dual of Lemma 3.1) if we can show:

(3.4) Whenever $X_{\alpha} \geqq C \cap A$ for $\alpha \in I$ (with $\left.\bar{I} \leqq \aleph\right)$ and $C \cup\left(\cap\left(X_{\beta} \mid \beta \in F\right)\right)=$ 1 for all finite $F \subset I$, then $C \cup\left(\cap\left(X_{a} \mid \alpha \in I\right)\right)=1$.

Since $C \cap A=b$ and $C=a \cup b$, (3.4) can be rewritten:

(3.4)' Whenever $X_{\alpha} \geqq b$ for $\alpha \in I($ with $\bar{I} \leqq \$)$ and $\alpha \cup\left(\cap\left(X_{\beta} \mid \beta \in F\right)\right)=$ $a \cup b \cup c$ for all finite $F \subset I$ then $a \cup\left(\cap\left(X_{\alpha} \mid \alpha \in I\right)\right)=a \cup b \cup c$.

Suppose that the hypotheses of (3.4)' hold and set $x_{a}=X_{\alpha} \cap(a \cup c)$. Then $x_{\alpha} \leqq a \cup c$ for all $\alpha$ and

$$
\begin{aligned}
& a \cup\left(\cap\left(x_{\beta} \mid \beta \in F\right)\right) \\
= & a \cup\left(\left(\cap\left(X_{\beta} \mid \beta \in F\right)\right) \cap(a \cup c)\right)=\left(a \cup\left(\cap\left(X_{\beta} \mid \beta \in F\right)\right)\right) \cap(a \cup c) \\
= & (a \cup b \cup c) \cap(a \cup c)=a \cup c .
\end{aligned}
$$

Since $l(a, c, \boldsymbol{N})$ holds, it follows that

$$
\begin{aligned}
& a \cup\left(\bigcap\left(x_{\alpha} \mid \alpha \in I\right)\right)=a \cup c ; a \cup\left(\cap\left(X_{\alpha} \mid \alpha \in I\right) \cap(a \cup c)\right)=a \cup c ; \\
& \left.a \cup\left(\bigcap\left(X_{\alpha} \mid \alpha \in I\right)\right) \geqq a \cup c \text { (hence }=a \cup b \cup c\right) .
\end{aligned}
$$

This means: (3.4)' does hold. This completes the proof of Lemma 3.5. 
Corollary 1. Suppose that $\left[0, a_{i} \cup a_{j}\right]$ is lower $\$$-complete for $i, j=1, \cdots, m$.

Suppose also that $l\left(a_{i}, a_{j}, \boldsymbol{W}\right)$ holds for all $i<j$. Then $\left[0, a_{1} \cup \cdots \cup a_{m}\right]$ is lower $\$$-complete.

Proof. This follows from Lemma 3.5 by induction on $m$, just as Corollary 1 to Lemma 3.1 followed from Lemma 3.1.

Corollary 2. Suppose that $\left[0, a_{i} \cup a_{j}\right]$ is lower $\aleph$-complete and lower $\boldsymbol{\aleph}$-continuous for $i, j=1, \cdots, m$. Then $\left[0, a_{1} \cup \cdots \cup a_{m}\right]$ is lower ઝi-continuous.

Proof. Since lower $\boldsymbol{\aleph}$-continuity of $\left[0, a_{i} \cup a_{j}\right]$ implies that $l\left(a_{i}, a_{j}, \boldsymbol{\aleph}\right)$ holds, Corollary 1 shows that $\left[0, a_{1} \cup \cdots \cup a_{m}\right]$ is lower $\boldsymbol{X}$-complete. The lower $\$$-continuity of $\left[0, a_{1} \cup \cdots \cup a_{m}\right]$ then follows from Lemma 3.4.

\section{Lemma 3.6. Suppose that}

(i) $a=a_{1} \cup a_{2} \cup \cdots \cup a_{m}$ for some finite $m \geqq 2$,

(ii) $a_{2} \sim a_{1}$,

(iii) $a_{i} \lesssim a_{1} \cup \cdots \cup a_{i-1}$ for $2<i \leqq m$,

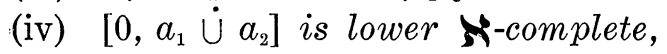

(v) $l\left(a_{1}, a_{2}, \lessgtr\right)$ holds.

Then $[0, a]$ is lower $\$$-complete.

Corollary. Suppose that (i), (ii), (iii) hold and also

(vi) $\left[0, a_{1} \dot{\cup} a_{2}\right]$ is lower $\aleph$-complete and lower $\aleph$-continuous.

Then $[0, a]$ is lower $\$$-complete and lower $\$$-continuous.

Proof. Lemma 3.6 and its Corollary follow from Lemma 3.5 and Lemma 3.4 just as Lemma 3.3 and its Corollary followed from Corollary 1 to Lemma 3.1 and [1, Theorem 4.3].

Theorem 3.1. Suppose that each of $\left[0, a_{i} \cup a_{j}\right]$ is an $\$$-von Neumanngeometry (respectively a von Neumann-geometry) for $i, j=1, \cdots, m$. Then $\left[0, a_{1} \cup \cdots \cup a_{m}\right]$ is an $\aleph$-von Neumann-geometry (respectively a von Neumann geometry).

Proof. This follows from Corollary 2 to Lemma 3.1 and Corollary 2 to Lemma 3.5.

COROLlaRY 1. Suppose that

(i) $a=a_{1} \cup a_{2} \cup \cdots \cup a_{m}$ for some finite $m \geqq 2$, 
(ii) $a_{2} \sim a_{1}$,

(iii) $a_{i} \lesssim a_{1} \cup \cdots \cup a_{i-1}$ for $2<i \leqq m$,

(iv) $\left[0, a_{1} \cup a_{2}\right]$ is an $\aleph$-von Neumann-geometry (respectively a von Neumann-geometry).

Then $[0, a]$ is an \$-von Neumann-geometry, respectively a von Neumanngeometry.

Proof. This follows from the Corollary to Lemma 3.3 and the Corollary to Lemma 3.6.

Corollary 2. Suppose that $\mathscr{R}$ is an $\boldsymbol{K}$-von Neumann-ring (respectively a von Neumann-ring). If $\bar{R}_{\mathscr{C}}$ has a basis $x_{1}, x_{2}, \cdots, x_{m}$ such that $x_{2} \sim x_{1}$ and $x_{i} \lesssim x_{1}$ for $2<i \leqq m$, then $\mathscr{R}_{2}$ is an $\aleph$-von Neumannring (respectively, a von Neumann-ring).

Proof. By hypothesis, the unit element of the lattice $\bar{R}_{\mathscr{R}}$ is the union $x_{1} \dot{\cup} \cdots \dot{U} x_{m}$. The unit element of $\bar{R}_{\mathscr{S}}$, with $\mathscr{S}=\mathscr{R}_{2}$, can be represented as a union $x_{1} \dot{\cup} \cdots \dot{U} x_{m} \dot{\cup} y_{1} \dot{\cup} \cdots \dot{U} y_{m}$ with $y_{i} \sim x_{i}$ and hence $y_{i} \lesssim x_{1}$ for $1 \leqq i \leqq m$. Since $\left[0, x_{1} \dot{\cup} x_{2}\right]$ is an $\aleph$-von Neumann geometry (respectively a von Neumann geometry) along with $\bar{R}_{\mathscr{S}}$, Corollary 1 applies and this completes the proof of Corollary 2.

Corollary 3. Suppose that $\mathscr{R}$ and $\mathscr{R}_{2}$ are both $\$$-von Neumannrings (respectively von Neumann-rings). Then $\mathscr{R}_{n}$ is an \$-von Neumann-ring (respectively a von Neumann-ring) for all finite $n$.

Proof. If $n>2$ the unit element of $\bar{R}_{\mathscr{S}}$, with $\mathscr{S}=\mathscr{R}_{n}$, can be expressed as $x_{1} \dot{U} x_{2} \dot{U} \cdots \dot{U} x_{n}$ where $x_{1}$ is the unit element of $\bar{R}_{\mathscr{R}}$, $x_{i} \sim x_{1}$ for all $i$, and $\left[0, x_{1} \dot{\cup} x_{2}\right]=\bar{R}_{\mathscr{R}_{2}}$. Theorem 3.1 applies and this completes the proof of Corollary 3 .

REMARK. Let $\mathscr{R}$ be the ring of sequences $x=\left(x^{n}\right)$ with all $x^{n}$ complex numbers and all but a finite number of $x^{n}$ real, with componentwise addition and multiplication; this example was given by Kaplansky [3, page 526]. This $\mathscr{R}$ is a von Neumann-ring but $\mathscr{R}_{2}$ is not even upper $\aleph_{0}$-complete.

Definition 3.1. If $L$ is a relatively complemented modular lattice, then an element $a$ is called Boolean (with respect to $L$ ) if $b_{1} \sim b_{2}, b_{1} \leqq a$ together imply $b_{1}=b_{2} ; a$ is called the Boolean part of $L$ (necessarily unique if it exists) $)^{2}$ if $a$ is Boolean and $a_{1} \leqq a$ for every Boolean $a_{1}$.

${ }^{2}$ This is an abuse of language: properly, $[0, a]$ should be called the Boolean part of $L$. 
Lemma 3.7. Suppose that $L$ is a relatively complemented modular lattice. If $(a, b) P$ holds then for every $c$ in $L, c \cap(a \cup b)=(c \cap a) \cup(c \cap b)$ and $[0, a \cup b]$ is the direct sum of $[0, a]$ and $[0, b]$. On the other hand if $a$ is Boolean then

(i) $b \leqq a$ implies that $b$ is Boolean,

(ii) $b \cap a=0$ implies that $(b, a) P$ holds,

(iii) $b \geqq a$ implies that the relative complement $[b-a]$ is unique,

(iv) $a \cap(b \cup c)=(a \cap b) \cup(a \cap c)$ for all $b, c$ in $L$,

(v) $[0, a]$ is a Boolean algebra.

Proof. Suppose that $(a, b) P$ holds and set $d=[(c \cap(a \cup b))-$ $((c \cap a) \cup(c \cap b))], d_{a}=(d \cup b) \cap a, d_{b}=(d \cup a) \cap b$. Then $d \leqq a \cup b$, $d \cap a=d \cap b=0, \quad d_{a} \dot{\cup} d=(d \cup b) \cap(d \cup a)=d_{b} \dot{\cup} d$, so $d_{a} \sim d_{b}$. Since $d_{a} \leqq a, d_{b} \leqq b$ and $(a, b) P$ holds, we must have: $d_{a}=0 ; b=d_{a} \cup b=$ $d \cup b ; d \leqq b$; hence $d=0, c \cap(a \cup b)=(c \cap a) \cup(c \cap b)$. If $c \leqq a \cup b$ then $c=(c \cap a) \cup(c \cap b)$; and if $c=c_{1} \cup c_{2}$ with $c_{1} \leqq a, c_{2} \leqq b$ then $c \cap a=c_{1} \cup\left(c_{2} \cap b \cap a\right)=c_{1} \cup 0=c_{1}, c \cap b=c_{2}$. This proves that $[0, a \cup b]$ is the direct sum of $[0, a]$ and $[0, b]$.

(i) and (ii) are obvious from the definition of Boolean element.

(ii) asserts that $a$ is in the centre of $L$ as defined in $[1,(2.5)]$. But if $a$ is in the centre of $L$ and $b$ is any element in $L$ with $b \geqq a$ then $a$ is in the centre of $[0, b]$, hence $[b-a]$ is uniquely determined (use $[1,(2.6)])$. This proves (iii).

If $b, c$ are arbitrary elements in $L$, set $b_{1}=[b-(a \cap b)], c_{1}=$

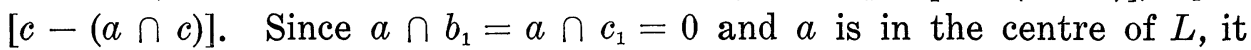
follows that $\left(a, b_{1}\right) P,\left(a, c_{1}\right) P$, hence $\left(a, b_{1} \cup c_{1}\right) P$ (use $\left.[1,(2,6)]\right)$; therefore $a \cap\left(b_{1} \cup c_{1}\right)=0$. By the modular law

$$
\begin{aligned}
a \cap(b \cup c) & =a \cap\left(b_{1} \cup c_{1} \cup(a \cap b) \cup(a \cap c)\right) \\
& =(a \cap b) \cup(a \cap c) \cup\left(a \cap\left(b_{1} \cup c_{1}\right)\right) \\
& =(a \cap b) \cup(a \cap c)
\end{aligned}
$$

and hence (iv) holds.

Thus $[0, a]$ is a distributive complemented lattice, equivalently: a Boolean algebra. This proves (v).

Lemma 3.8. Suppose that $L$ has a unit element $1=a_{1} \cup a_{2} \cup \cdots \cup a_{m}$ with $m \geqq 2, a_{2} \sim a_{1}, a_{i} \lesssim a_{1}$ for $2<i \leqq m$ and $a_{1} \cap a_{2}=0$. Then the Boolean part of $L$ exists and is 0 .

Proof. By Lemma 3.2 we may assume that $1=a_{1} \dot{U} \cdots \dot{U} a_{m}$ with $m \geqq 2, a_{2} \sim a_{1}$ and $a_{i} \lesssim a_{1}$ for $2<i \leqq m$.

To prove Lemma 3.8 we may suppose that $a \neq 0$ and we need only exhibit elements $b_{1}, b_{2}$ such that $b_{1} \leqq a, b_{1} \sim b_{2}$, and $b_{1} \neq b_{2}$. 
If $a_{i} \cap a \neq 0$ for any $i$ it suffices to choose this element as $b_{1}$ since the relations $a_{1} \sim a_{2}$ and $a_{i} \lesssim a_{1}$ if $i \neq 1$ imply $b_{1} \sim b_{2}$ for some $b_{2} \neq b_{1}$ (even $b_{1} \cap b_{2}=0$ ).

On the other hand, if $a_{i} \cap a=0$ for all $i$, set $b_{1}=\left(a_{1} \cup \cdots \cup a_{i}\right) \cap a$ where $i$ is the smallest integer for which this element is different from 0 (necessarily $1<i \leqq m)$ and set $b_{2}=\left(\left(a_{1} \cup \cdots \cup a_{i-1}\right) \dot{\cup} b_{1}\right) \cap a_{i}$. Then $b_{1} \sim b_{2}$ since $\left(a_{1} \cup \cdots \cup a_{i-1}\right) \dot{\cup} b_{1}=\left(a_{1} \cup \cdots \cup a_{i-1}\right) \cup b_{2}$; and $b_{1} \neq b_{2}$ since $b_{2} \leqq a_{i}$ and $b_{1} \cap a_{i} \leqq a \cap a_{i}=0$. This completes the proof of Lemma 3.8.

Lemma 3.9. Suppose that $L$ is an upper complete complemented modular lattice and let $a$ be the union of all Boolean elements in $L$. Then $a$ is the Boolean part of $L$.

Proof. We need only show that $a$ is Boolean, that is, we may suppose that $b \leqq a$, that $\varphi$ is a perspective mapping of $[0, b]$, that $b \neq \varphi(b)$ and we need only derive a contradiction. By replacing $b$ by $[b-(b \cap \varphi(b))]$ we may suppose $b \neq 0$ and $b \cap \varphi(b)=0$.

Now for every $c:(\varphi(b \cap c)) \sim(b \cap c)$ and $(\varphi(b \cap c)) \cap(b \cap c)=0$. If $c$ is Boolean this implies: $b \cap c=0$, and hence (since $c$ is Boolean) $(b, c) P$ holds. It follows from [1, formula $(2.6)]$ that $(b, a) P$ holds, contradicting the fact that $b \neq 0$ and $b \leqq a$. This contradiction proves Lemma 3.9 .

THEOREM 3.2. Suppose that $L$ is a relatively complemented modular lattice and

(i) $\quad a=a_{0} \cup a_{1} \cup a_{2} \cup \cdots \cup a_{m}$ for some finite $m \geqq 2$,

(ii) $\left(a_{0}, a_{1} \cup \cdots \cup a_{m}\right) P$ holds,

(iii) $a_{2} \sim a_{1}, a_{2} \cap a_{1}=0$,

(iv) $a_{i} \lesssim a_{1} \cup \cdots \cup a_{i-1}$ for $2<i \leqq m$,

(v) $\varphi$ is a perspective mapping of $[0, b]$ with $\varphi(b) \leqq a$.

Let $\pi$ denote one of the properties: to be upper $\aleph$-complete and upper $\boldsymbol{\aleph}$-continuous, or to be lower $\boldsymbol{\aleph}$-complete and lower $\boldsymbol{\aleph}$-continuous. Then $[0, a \cup b]$ has property $\pi$ if both of $\left[0, a_{1} \cup a_{2}\right]$ and $\left[0, a_{0} \cup \varphi^{-1}\left(a_{0} \cap \varphi(b)\right)\right]$ have property $\pi$; if $a_{0}$ is the Boolean part of $[0, a]$ and $[0, b]$ has $a$ Boolean part $b_{0}$, it is sufficient that $\left[0, a_{1} \cup a_{2}\right]$ and $\left[0, a_{0} \cup b_{0}\right]$ should both have property $\pi$.

Proof. Since $\left(a_{0}, a_{1} \cup \cdots \cup a_{m}\right) P$ holds, Lemma 3.7 shows that $\varphi(b)=\varphi\left(b_{1}\right) \dot{\cup} \varphi\left(b_{2}\right)$ where $b_{1}=\varphi^{-1}\left(a_{0} \cap \varphi(b)\right)$ and $b_{2}=\varphi^{-1}\left(\left(a_{1} \cup \cdots \cup a_{m}\right) \cap \varphi(b)\right)$. Then $\left(a_{0} \cup b_{1}, a_{1} \cup \cdots \cup a_{m} \cup b_{2}\right) P$ holds (use [1, (2.6)]).

By Lemma 3.7, $[0, a \cup b]$ is the direct sum of $\left[0, a_{0} \cup b_{1}\right]$ and $\left[0, a_{1} \cup \cdots \cup a_{m} \cup b_{2}\right]$ and has property $\pi$ if each of the summands has it. 
Since $b_{2} \lesssim a_{1} \cup \cdots \cup a_{m},\left[0, a_{1} \cup \cdots \cup a_{m} \cup b_{2}\right]$ has property $\pi$ if $\left[0, a_{1} \cup a_{2}\right]$ has it, by Lemma 3.3 and its Corollary and Lemma 3.6 and its Corollary.

If $a_{0}$ is the Boolean part of $[0, a]$ then $\varphi(b) \cap a_{0}$ is Boolean with respect to $[0, a]$, a fortiori Boolean with respect to $[0, \varphi(b)]$. Thus, $b_{1}$ is Boolean with respect to $[0, b]$. If $[0, b]$ has a Boolean part $b_{0}$ then $b_{1} \leqq b_{0}$ and $a_{0} \cup b_{1} \leqq a_{0} \cup b_{0}$, hence $\left[0, a_{0} \cup b_{1}\right]$ has property $\pi$ if $\left[0, a_{0} \cup b_{0}\right]$ has it.

This proves all parts of Theorem 3.2.

REMARK. If $\mathscr{R}$ is a von Neumann ring then $\mathscr{R}$ has a unique decomposition as a direct sum $\mathscr{R}=\mathscr{B} \oplus \mathscr{R}$ such that $\bar{R}_{\mathscr{B}}$ is the Boolean part of $\bar{R}_{\mathscr{O}}$ and $\bar{R}_{\mathscr{B}}$ has a basis $x_{1}, x_{2}, x_{3}$ with $x_{2} \sim x_{1}$ and $x_{3} \lesssim x_{1}$. Then Theorem 3.2 and Corollary 2 to Theorem 3.1 apply and show that $\mathscr{R}_{2}$ is a von Neumann ring if and only if $\mathscr{B}_{2}$ is a von Neumann ring (for details see [2]).

\section{REFERENCES}

1. Ichiro Amemiya and Israel Halperin, Complemented modular lattices, Canadian J. of Math., 11 (1959), 481-520.

2. Israel Halperin, Elementary divisors in von Neumann rings, Acta Scientiarum Mathematicarum Szeged, to appear.

3. Irving Kaplansky, Any orthocomplemented complete modular lattice is a continuous geometry, Annals of Math., 61. (1955), 524-541.

4. John von Neumann, Continuous Geometry, Princeton University Press, 1960.

QUEEN'S UNIVERSITY,

Kingston, CANADA. 



\section{PACIFIC JOURNAL OF MATHEMATICS}

\section{EDITORS}

\author{
Ralph S. Phillips \\ Stanford University \\ Stanford, California \\ M. G. Arsove \\ University of Washington \\ Seattle 5 , Washington
}

\author{
A. L. Whiteman \\ University of Southern California \\ Los Angeles 7, California \\ Lowell J. Paige \\ University of California \\ Los Angeles 24, California
}
E. F. BECKENBACH
D. DERRY
ASSOCIATE EDITORS
T. M. CHERRY
M. OHTSUKA
H. L. ROYDEN
E. G. STRAUS
E. SPANIER
F. WOLF

\section{SUPPORTING INSTITUTIONS}

\author{
UNIVERSITY OF BRITISH COLUMBIA \\ CALIFORNIA INSTITUTE OF TECHNOLOGY \\ UNIVERSITY OF CALIFORNIA \\ MONTANA STATE UNIVERSITY \\ UNIVERSITY OF NEVADA \\ NEW MEXICO STATE UNIVERSITY \\ OREGON STATE UNIVERSITY \\ UNIVERSITY OF OREGON \\ OSAKA UNIVERSITY \\ UNIVERSITY OF SOUTHERN CALIFORNIA
}

\author{
STANFORD UNIVERSITY \\ UNIVERSITY OF TOKYO \\ UNIVERSITY OF UTAH \\ WASHINGTON STATE UNIVERSITY \\ UNIVERSITY OF WASHINGTON \\ AMERICAN MATHEMATICAL SOCIETY \\ CALIFORNIA RESEARCH CORPORATION \\ SPACE TECHNOLOGY LABORATORIES \\ NAVAL ORDNANCE TEST STATION
}

Mathematical papers intended for publication in the Pacific Journal of Mathematıcs should be typewritten (double spaced), and the author should keep a complete copy. Manuscripts may be sent to any one of the four editors. All other communications to the editors should be addressed to the managing editor, L. J. Paige at the University of California, Los Angeles 24, California.

50 reprints per author of each article are furnished free of charge; additional copies may be obtained at cost in multiples of 50 .

The Pacific Journal of Mathematics is published quarterly, in March, June, September, and December. Effective with Volume 13 the price per volume (4 numbers) is $\$ 18.00$; single issues, $\$ 5.00$. Special price for current issues to individual faculty members of supporting institutions and to individual members of the American Mathematical Society: $\$ 8.00$ per volume; single issues $\$ 2.50$. Back numbers are available.

Subscriptions, orders for back numbers, and changes of address should be sent to Pacific Journal of Mathematics, 103 Highland Boulevard, Berkeley 8, California.

Printed at Kokusai Bunken Insatsusha (International Academic Printing Co., Ltd.), No. 6, 2-chome, Fujimi-cho, Chiyoda-ku, Tokyo, Japan.

PUBLISHED BY PACIFIC JOURNAL OF MATHEMATICS, A NON-PROFIT CORPORATION

The Supporting Institutions listed above contribute to the cost of publication of this Journal, but they are not owners or publishers and have no responsibility for its content or policies. 


\section{Pacific Journal of Mathematics}

\section{Vol. 12 , No. 4 \\ April, 1962}

Tsuyoshi Andô, On fundamental properties of a Banach space with a cone ..... . 1163

Sterling K. Berberian, A note on hyponormal operators ................ 1171

Errett Albert Bishop, Analytic functions with values in a Frechet space . . . . . . . 1177

(Sherman) Elwood Bohn, Equicontinuity of solutions of a quasi-linear equation ............................................ 1193

Andrew Michael Bruckner and E. Ostrow, Some function classes related to the class of convex functions . . . . . . . . . . . . . . . . . . . . . . . . 1203

J. H. Curtiss, Limits and bounds for divided differences on a Jordan curve in the complex domain . . ................................. 1217

P. H. Doyle, III and John Gilbert Hocking, Dimensional invertibility . . . . . . . . 1235

David G. Feingold and Richard Steven Varga, Block diagonally dominant matrices and generalizations of the Gerschgorin circle theorem ................. 1241

Leonard Dubois Fountain and Lloyd Kenneth Jackson, A generalized solution of the boundary value problem for $y^{\prime \prime}=f\left(x, y, y^{\prime}\right) \ldots \ldots \ldots \ldots \ldots \ldots \ldots \ldots \ldots$

Robert William Gilmer, Jr., Rings in which semi-primary ideals are primary. . . . . 1273

Ruth Goodman, K-polar polynomials .......................... 1277

Israel Halperin and Maria Wonenburger, On the additivity of lattice completeness ........................................... 1289

Robert Winship Heath, Arc-wise connectedness in semi-metric spaces . . . . . . . . 1301

Isidore Heller and Alan Jerome Hoffman, On unimodular matrices . . . . . . . . . . . 1321

Robert G. Heyneman, Duality in general ergodic theory . . . . . . . . . . . . . . . 1329

Charles Ray Hobby, Abelian subgroups of p-groups . . . . . . . . . . . . . . . 1343

Kenneth Myron Hoffman and Hugo Rossi, The minimum boundary for an analytic

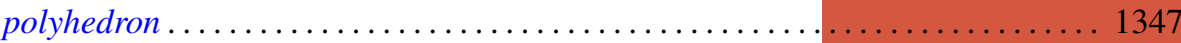

Adam Koranyi, The Bergman kernel function for tubes over convex cones ........ 1355

Pesi Rustom Masani and Jack Max Robertson, The time-domain analysis of a continuous parameter weakly stationary stochastic proces.

William Schumacher Massey, Non-existence of almost-complex structures on

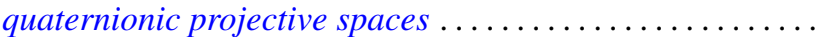

Deane Montgomery and Chung-Tao Yang, A theorem on the action of $\mathrm{SO}(3) \ldots . .1385$

Ronald John Nunke, A note on Abelian group extensions . . . . . . . . . . . . . 1401

Carl Mark Pearcy, A complete set of unitary invariants for operators generating finite $W^{*}$-algebras of type $I$

Edward C. Posner, Integral closure of rings of solutions of linear differential equations.

Duane Sather, Asymptotics. III. Stationary phase for two parameters with an application to Bessel functions.

J. Śladkowska, Bounds of analytic functions of two complex variables in domains

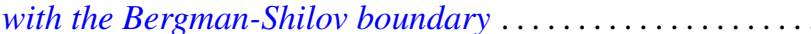

Joseph Gail Stampfli, Hyponormal operators .

George Gustave Weill, Some extremal properties of linear combinations of kernels

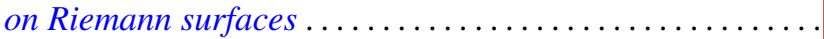

